Ferrata Storti Foundation

\title{
Downregulation of TREM-like transcript-1 and collagen receptor $\alpha 2$ subunit, two novel RUNX1-targets, contributes to platelet dysfunction in familial platelet disorder with predisposition to acute myelogenous leukemia
}

Haematologica 2019

Volume 104(6):1244-1255

\section{Correspondence:}

PAULA HELLER

paulaheller@hotmail.com

HANA RASLOVA

hana.raslova@gustaveroussy.fr

Received: January 20, 2018.

Accepted: December 10, 2018.

Pre-published: December 13, 2018.

doi:10.3324/haematol.2018.188904

Check the online version for the most updated information on this article, online supplements, and information on authorship \& disclosures: www.haematologica.org/content/104/6/1244

(C)2019 Ferrata Storti Foundation

Material published in Haematologica is covered by copyright. All rights are reserved to the Ferrata Storti Foundation. Use of published material is allowed under the following terms and conditions:

https://creativecommons.org/licenses/by-nc/4.0/legalcode. Copies of published material are allowed for personal or internal use. Sharing published material for non-commercial purposes is subject to the following conditions:

https://creativecommons.org/licenses/by-nc/4.0/legalcode, sect. 3. Reproducing and sharing published material for commercial purposes is not allowed without permission in writing from the publisher.
Ana C. Glembotsky, ${ }^{1,2^{*}}$ Dominika Sliwa, ${ }^{3^{*}}$ Dominique Bluteau, ${ }^{3,4^{*}}$ Nathalie Balayn, ${ }^{3}$ Cecilia P. MarinOyarzún, ${ }^{1,2}$ Anna Raimbault, ${ }^{3}$ Marie Bordas, ${ }^{3}$ Nathalie Droin, ${ }^{3,5}$ Iryna Pirozhkova, ${ }^{6}$ Valance Washington, ${ }^{7}$ Nora P. Goette, ${ }^{1}$ Rosana F. Marta,,$^{1,2}$ Rémi Favier, ${ }^{3,8}$ Hana Raslova, ${ }^{3^{* \star}}$ and Paula G. Heller ${ }^{1,2^{\star *}}$

${ }^{1}$ Instituto de Investigaciones Médicas Alfredo Lanari, Facultad de Medicina, Universidad de Buenos Aires, Buenos Aires, Argentina; ${ }^{2}$ Hematología Investigación, Consejo Nacional de Investigaciones Científicas y Tecnológicas (CONICET), Universidad de Buenos Aires, Instituto de Investigaciones Médicas (IDIM), Buenos Aires, Argentina; ${ }^{3}$ INSERM UMR 1170, Gustave Roussy, Université Paris-Saclay, Equipe Labellisée par la Ligue Nationale Contre le Cancer, Villejuif, France; ${ }^{4}$ Ecole Pratique des Hautes Etudes (EPHE), Paris, France; ${ }^{5}$ Gustave Roussy, Université Paris-Saclay, Genomic Platform UMS AMMICA, Villejuif, France; ${ }^{6}$ CNRS UMR 8126, Gustave Roussy, Université Paris-Saclay, Villejuif, France; ' ${ }^{7}$ Department of Biology, University of Puerto Rico-Rio Piedras, San Juan, Puerto Rico and ${ }^{8}$ Assistance Publique-Hôpitaux de Paris, Hôpital Trousseau, CRPP, Services d'Hématologie Biologique et Clinique, Paris, France

${ }^{\star} A C G, D S$ and $D B$ contributed equally to this work. ${ }^{*} H R$ and $P G H$ contributed equally to this work as senior co-authors.

\section{ABSTRACT}

$\checkmark$ ermline RUNX1 mutations lead to thrombocytopenia and platelet dysfunction in familial platelet disorder with predisposition to acute myelogenous leukemia (AML). Multiple aspects of platelet function are impaired in these patients, associated with altered expression of genes regulated by RUNX1. We aimed to identify RUNX1targets involved in platelet function by combining transcriptome analysis of patient and shRUNX1-transduced megakaryocytes (MK). Down-regulated genes included TREM-like transcript (TLT)-1 (TREML1) and the integrin subunit alpha ( $\alpha$ )-2 (ITGA2) of collagen receptor $\alpha 2$-beta ( $\beta$ )-1, which are involved in platelet aggregation and adhesion, respectively. RUNX1 binding to regions enriched for H3K27Ac marks was demonstrated for both genes using chromatin immunoprecipitation. Cloning of these regions upstream of the respective promoters in lentivirus allowing mCherry reporter expression showed that RUNX1 positively regulates TREML1 and ITGA2, and this regulation was abrogated after deletion of RUNX1 sites. TLT-1 content was reduced in patient MK and platelets. A blocking anti-TLT-1 antibody was able to block aggregation of normal but not patient platelets, whereas recombinant soluble TLT-1 potentiated fibrinogen binding to patient platelets, pointing to a role for TLT-1 deficiency in the platelet function defect. Low levels of $\alpha 2$ integrin subunit were demonstrated in patient platelets and $M K$, coupled with reduced platelet and $\mathrm{MK}$ adhesion to collagen, both under static and flow conditions. In conclusion, we show that gene expression profiling of RUNX1 knock-down or mutated MK provides a suitable approach to identify novel RUNX1 targets, among which downregulation of TREML1 and ITGA2 clearly contribute to the platelet phenotype of familial platelet disorder with predisposition to AML. 


\section{Introduction}

The transcription factor RUNX1 is a key regulator of the megakaryocytic lineage, where it participates in a complex transcriptional network co-ordinating platelet biogenesis and function. ${ }^{1,2}$ RUNX1 co-operates with other transcriptional regulators, including GATA-1, FLI-1 and SCL at megakaryocyte (MK)-specific promoters, ${ }^{1}$ whereas co-occupancy of RUNX1 with FLI-1 and NF-E2 has been shown to prime the late MK program. ${ }^{3}$ Conditional RUNX1 inactivation in mice leads to MK maturation arrest and a substantial decline in platelet counts, highlighting the key role of RUNX1 as a master regulator of the MK lineage. ${ }^{4}$ Germline RUNX1 mutations in humans underlie familial platelet disorder with predisposition to acute myelogenous leukemia (FPD/AML), which is characterized by thrombocytopenia, platelet dysfunction, and a lifelong $30-50 \%$ predisposition to hematologic malignancies, including myeloid and lymphoid neoplasms. ${ }^{5}$ RUNX1 mutations exerting dominant-negative effects over the wild-type (WT) protein are associated with a higher leukemic rate than those acting via haploinsufficiency, ${ }^{6}$ whereas no differences in the severity of the platelet phenotype are seen between both types of mutations. ${ }^{7}$ Although once considered a rare condition, FPD/AML is now diagnosed at increasing frequency due to heightened diagnostic awareness during the workup of individuals presenting with thrombocytopenia of uncertain etiology or hereditary myeloid malignancies.

The platelet defect in FPD/AML is complex and includes abnormalities in platelet number and function, which lead to a bleeding diathesis of variable severity, ranging from mild or asymptomatic cases to a severe bleeding tendency. Thrombocytopenia is usually mild to moderate and is caused by impaired platelet production secondary to defects in multiple steps of MK development, including MK differentiation, maturation, polyploidization and proplatelet formation. ${ }^{2}$ While marked dysmegakaryopoiesis with a severe defect in proplatelet formation is observed in vitro, the presence of only mild thrombocytopenia, often at the lower limit of normal range, suggests a yet unknown compensatory mechanism in vivo. The platelet function defect is present in most, if not all, patients with FPD/AML and involves multiple abnormalities in platelet structure and activation pathways, including defective platelet aggregation and release, dense granule deficiency, associated in some pedigrees with partial alpha ( $\alpha$ )-granule defect, and impaired $\alpha$ IIb-beta ( $\beta$ )-3 (GPIIbIIIa) activation and outside-in signaling. ${ }^{8,9}$ These abnormalities are likely due to altered expression of RUNX1-targets involved in platelet biology. The study of FPD/AML platelet samples has revealed downregulation of several RUNX1-regulated genes. Transcriptome analysis of platelets from one patient showed reduced levels of MYL9, ALOX12, PKC $\theta$, RAB1B and PLDN, ${ }^{10}$ whereas dysregulated expression of MPL, ${ }^{11}$ MYH10 12 , RAB27 ${ }^{8}$ and NF-E $2^{8}$ has been identified by a candidate-gene approach in other pedigrees. However, the mechanisms underlying FPD/AML platelet function defect and the effects of RUNX1 mutations on the expression of other potential genes are still not completely understood. In this study, we combined expression profiling of mature shRUNX1-transduced and FPD/AML MK to gain further insight into RUNX1-regulated genes involved in platelet function. Using this approach, we identified triggering receptor expressed on myeloid cells (TREM)-like transcript (TLT)-1 and integrin subunit $\alpha 2$ of collagen receptor $\alpha 2 \beta 1$ as two novel RUNX-1 targets, whose expression was decreased in FPD/AML MK and platelets.

\section{Methods}

\section{Human samples}

Patients from three previously described FPD/AML pedigrees $^{2,8,11}$ (Table 1 and Online Supplementary Table S1), healthy subjects, and individuals after stem cell mobilization were included. At the time of the study, patients had thrombocytopenia and/or platelet dysfunction with no evidence of myelodysplastic or leukemic transformation. Details on experiments performed on each patient are provided in Online Supplementary Table S2. The study was approved by the Ethics Committee of INSERM RBM 01-14 for the project "Network on the inherited diseases of platelet function and platelet production" in France and the Ethics Committee of the Instituto de Investigaciones Médicas "Dr. Alfredo Lanari" in Argentina. Patients and controls gave signed informed consent.

\section{Megakaryocyte culture and transcriptome analysis}

CD34 $4^{+}$cells were isolated from cord blood, leukapheresis samples or peripheral blood of patients and healthy subjects using a magnetic cell-sorting system (AutoMACS or MiniMACS, Miltenyi Biotec SAS, Paris, France) and grown in serum free medium ${ }^{2}$ or Stem Span medium (StemCell Technologies, Vancouver, BC, Canada), supplemented with 10 $\mathrm{ng} / \mathrm{mL}$ thrombopoietin (TPO) (Kirin Brewery, Tokyo, Japan or Miltenyi Biotec) and $25 \mathrm{ng} / \mathrm{mL}$ Stem Cell Factor (SCF) (Biovitrum AB, Stockholm, Sweden or Miltenyi Biotec). For culture of patient MK, $10 \mathrm{ng} / \mathrm{mL}$ IL-6 (Tebu or Miltenyi Biotec), 100 U/mL IL-3 (Novartis or R\&D Systems, MN, USA) and $1 \mathrm{ng} / \mathrm{mL}$ fetal liver tyrosine kinase 3 ligand (FLT3-L) (Celldex Therapeutics or R\&D Systems) were added.

For transcriptome analysis, patient and control MK were cultured as detailed above, stained on day 10 of culture with allophycocyanin (APC)-conjugated anti-CD41 and phycoerythrin (PE)-anti-CD42 antibodies (BD Biosciences, Le Pont de Claix, France), and $\mathrm{CD} 41^{+} \mathrm{CD} 42^{+}$were sorted by flow cytometry. CD34 $4^{+}$cells from leukapheresis samples were transduced on days 6 and 7 of culture with lentiviruses encoding shRUNX1_1, shRUNX1_2 and shSCR (control shRNA), and $\mathrm{CD} 41^{+} \mathrm{CD} 42^{+} \mathrm{GFP}^{+}$cells were sorted on day 10 by flow cytometry, as previously described. ${ }^{2,13}$ RNA was extracted using the RNeasy Micro Kit (Qiagen, France) according to the manufacturer's instructions. Transcriptome analysis was performed using the Agilent Whole Human Genome Microarray (see Online Supplementary Methods).

\section{Statistical analysis}

For comparison between patients and controls, MannWhitney test or Wilcoxon matched pairs test were applied. For promoter activity and chromatin immunoprecipitation-polymerase chain reaction (ChIP-PCR) assays, paired $t$-test was used. For assessment of the effect of a blocking anti-TLT-1 antibody on proplatelet formation, repeated measures ANOVA was used. All statistical analyses were two-sided; $P<0.05$ was considered significant. The GraphPad Prism 6.01 (La Jolla, CA, USA) software was used for analysis.

Other methods are described in the Online Supplementary Appendix. 
A Patient MKs signature up-regulated genes

shRUNX1 MKs signature up-regulated genes

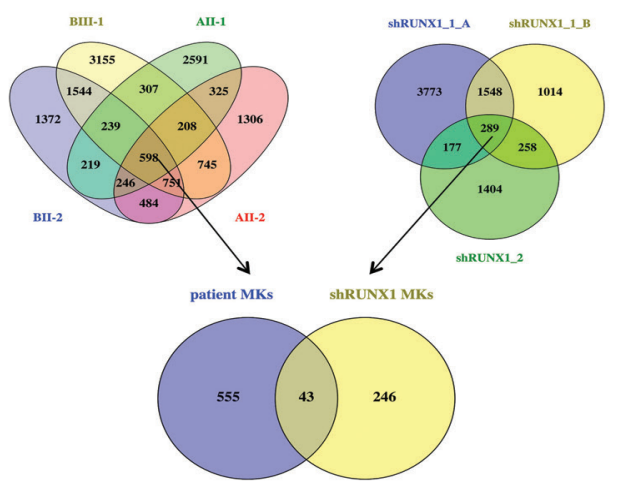

C

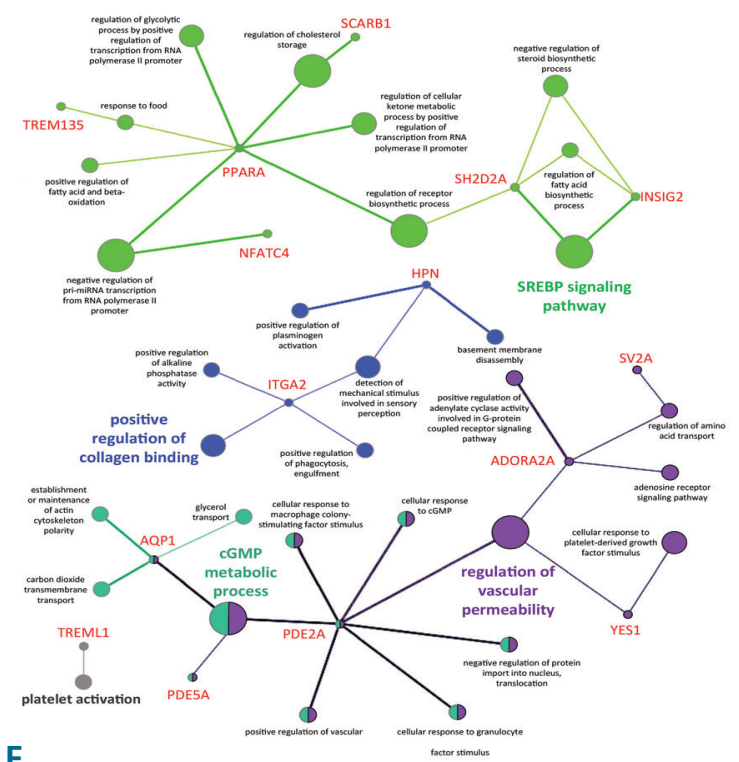

F

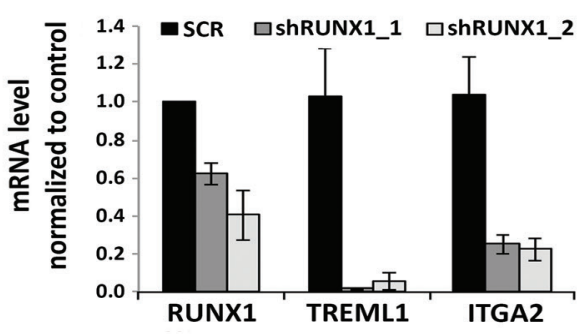

B Patient MKs signature down-regulated genes

shRUNX1 MKs signature down-regulated genes

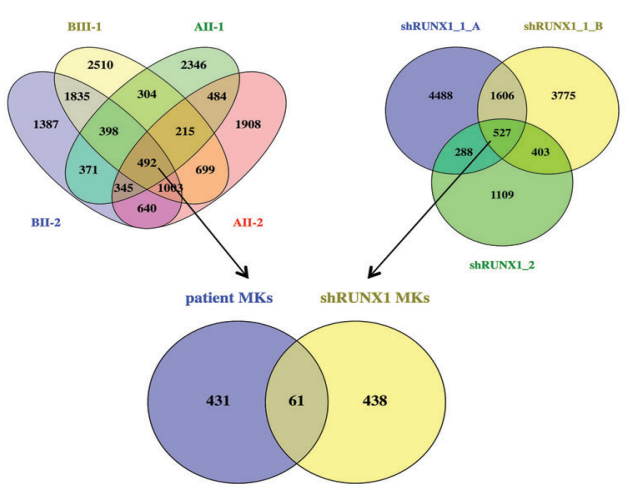

D

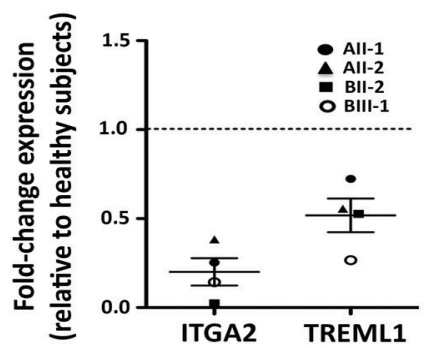

E

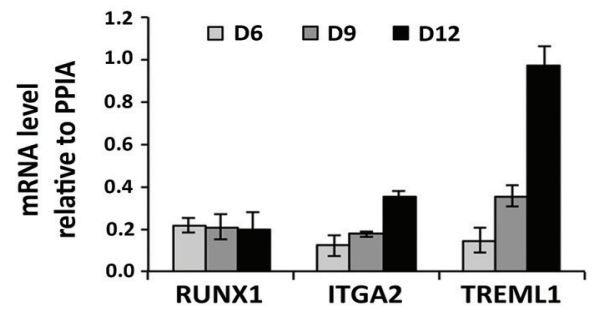

G

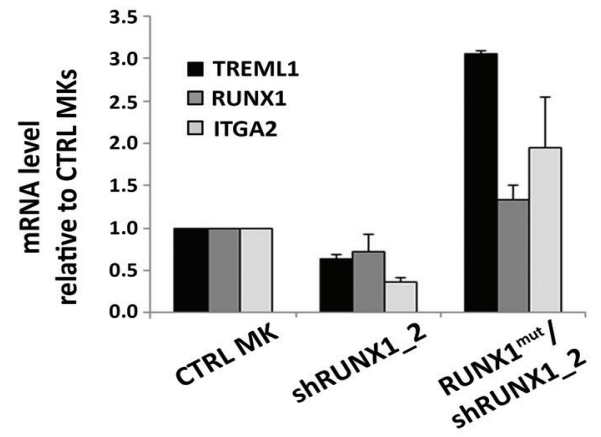

Figure 1. Transcriptome of RUNX1-deficient megakaryocytes (MK) reveals downregulation of TREML1 and ITGA2. (A and B) Venn diagram representation of the overlap between genes deregulated in patient MK (BII-2, BIII-1, All-1 and All-2), between genes deregulated in shRUNX1-transduced MK (shRUNX1_1_A, shRUNX1_1_B and shRUNX1_2), and between genes deregulated in both patient and shRUNX1-transduced MK. (A) A total of 598 genes were up-regulated in patient MK $(n=4)$ and 289 genes were up-regulated in shRUNX1-transduced MK $(n=3)$. A total of 43 genes were found to be up-regulated in both patient and shRUNX1transduced MK. (B) A total of 492 genes were down-regulated in patient MK $(n=4)$ and 499 genes $(527$ probes) were down-regulated in shRUNX1-transduced MK $(n=3)$. A total of 61 genes were found to be down-regulated in both patient and shRUNX1-transduced MK. shRUNX1_1 was used in two experiments (shRUNX1_1_A and shRUNX1_1_B) and shRUNX_2 in one. (C) Differentially down-regulated genes and functions associated with different biological processes. Gene ontology (GO) terms and differentially down-regulated genes are represented as nodes based on their kappa score $>0.4$. The node size represents the G0 terms enrichment significance. (D) Microarray data for ITGA2 and TREML1 in MK from patients (All-1 and All-2, carrying the R174Q mutation, and BII-2 and BIII-1, with the R139X mutation) relative to healthy subjects $(\mathrm{n}=4)$ (horizontal dashed line). (E) Real-time polymerase chain reaction (RT-PCR) analysis of RUNX1, ITGA2 and TREML1 transcript levels normalized to PPIA during normal MK in vitro differentiation. Cells were analyzed on day (D)6, D9, and D13 of culture. Data represent Mean \pm Standard Deviation (SD) of three independent experiments. (F) RT-PCR analysis of RUNX1, TREML1 and ITGA2 transcript levels normalized to PPIA in MK transduced with lentiviruses encoding shRUNX1 (shRUNX1_1 and shRUNX_2) relative to control shRNA (shSCR). Data represent Mean \pm SD of three independent experiments. (G) RTPCR analysis of RUNX1, TREML1 and ITGA2 transcript levels normalized to HPRT and relative to control (CTRL) MK. CTRL MK: MK transduced with control lentivirus expressing Cherry and lentivirus expressing shSCR-GFP; shRUNX1_2: MK transduced with control lenvtivirus expressing Cherry and lentivirus expressing shRUNX1_2 and GFP; RUNX1mut/shRUNX1_2: MK transduced with lentivirus expressing RUNX1 ${ }^{\text {mut }}$ and Cherry and with lentivirus expressing shRUNX1_2 and GFP. RUNX1 ${ }^{\text {mut }}$ : WT RUNX1 cDNA was cloned into the lentivirus pRRL_EF1a_MCS/PGK-Cherry and mutation in four nucleotides, keeping the same aminoacids, was introduced to avoid recognition of the cDNA by shRUNX1_2. Data represent Mean $\pm S D(n=2)$. 
Table 1. Features of familial platelet disorder with predisposition to acute myelogenous leukemia patients.

\begin{tabular}{|c|c|c|c|c|c|c|c|c|c|c|}
\hline \multirow{2}{*}{$\begin{array}{l}\text { Pedigree/ } \\
\text { patient }\end{array}$} & \multirow{2}{*}{$\begin{array}{l}\text { Sex/Age } \\
\text { (years) }\end{array}$} & \multirow{2}{*}{$\begin{array}{l}\text { Platelet count } \\
\left(\times 10^{\circ} / \text { L) }\right.\end{array}$} & \multirow{2}{*}{$\begin{array}{l}\text { MPV } \\
\text { (fiL) }\end{array}$} & \multirow{2}{*}{$\begin{array}{l}\text { ISTH-BAT } \\
\text { (score) }\end{array}$} & \multicolumn{4}{|c|}{ Platelet aggregation (\%) } & \multirow{2}{*}{$\begin{array}{l}\text { RUNX1 } \\
\text { mutation }\end{array}$} & \multirow{2}{*}{$\begin{array}{c}\text { RUNX1 } \\
\text { RNA levels }\end{array}$} \\
\hline & & & & & ADP & EPI & COL & AA & & \\
\hline AII-1 & $\mathrm{M} / 38$ & 90 & 9.6 & 1 & $55 \pm 7$ & $60 \pm 7$ & $40 \pm 4$ & $60 \pm 5$ & p.R174Q & $1.13^{*}$ \\
\hline AII-2 & $\mathrm{F} / 39$ & 116 & 10.6 & 2 & $37 \pm 2$ & $65 \pm 9$ & $60 \pm 20$ & $70 \pm 10$ & p.R174Q & $0.92 *$ \\
\hline BII-2 & $\mathrm{F} / 37$ & 130 & 9 & 1 & $24 \pm 4$ & $8 \pm 4$ & $33 \pm 3$ & $53 \pm 10$ & p.R139X & $0.46^{*}$ \\
\hline BIII-1 & $\mathrm{M} / 2$ & 106 & 10.2 & 2 & $40 \pm 10$ & $12 \pm 5$ & $28 \pm 4$ & $28 \pm 4$ & p.R139X & $2.66^{*}$ \\
\hline DII-1 & $\mathrm{F} / 51$ & 145 & 11.3 & 5 & $31 \pm 2$ & $14 \pm 6$ & $20 \pm 0$ & $29 \pm 40$ & p.T219Rfs*8 & $3.2^{* *}$ \\
\hline DIII-1 & $\mathrm{M} / 30$ & 115 & 10.5 & 6 & $30 \pm 3$ & $15 \pm 0$ & $8 \pm 7$ & $4 \pm 6$ & p.T219Rfs*8 & $2.9^{* *}$ \\
\hline DIII-3 & $\mathrm{F} / 27$ & 94 & 10.4 & 4 & $34 \pm 9$ & $19 \pm 2$ & $21 \pm 6$ & $34 \pm 41$ & p.T219Rfs*8 & $2.4^{* *}$ \\
\hline
\end{tabular}

MPV: mean platelet volume; ISTH-BAT: International Society on Thrombosis and Hemostasis-bleeding assessment tool; ADP: adenosine diphosphate;:EPI: epinephrine; COL: collagen; AA: arachidonic acid; nd: not done. Pedigrees $\mathrm{A}^{2}{ }^{2} \mathrm{~B}^{2}$ and $\mathrm{D}^{8,11}$ were previously described. Reference values for MPV, 8.9-12.5 fL. Platelet aggregation in response to $2 \mu \mathrm{M}$ ADP, $1 \mu \mathrm{M}$ epinephrine, $4 \mu \mathrm{g} / \mathrm{mL}$ collagen, and $1 \mathrm{mM}$ arachidonic acid was performed as described, ${ }^{8,9}$ and expressed as maximal light transmission percentage; Mean \pm Standard Deviation values are shown; reference values, $75 \pm 9,78 \pm 9.5,77 \pm 11$ and $79 \pm 9 \%$, respectively. ${ }^{*}$ Microarray data in patient megakaryocytes; * * quantitative polymerase chain reaction data in patient platelets, relative to healthy subjects $(n=4)$, set as 1 .

\section{Results}

Gene expression profiling of shRUNX1-transduced and familial platelet disorder with predisposition to acute myelogenous leukemia megakaryocytes reveals downregulation of TREML1 and ITGA2

To identify RUNX1-targets that could be involved in FPD/AML platelet dysfunction, we first performed transcriptome analysis of mature $\left(\mathrm{CD} 41^{+} \mathrm{CD} 42^{+}\right) \mathrm{MK}$ cultured from four patients, two carrying the $R 174 Q$ mutation, and two with the $R 139 X$ mutation. We then analyzed the transcriptome of MK cultured from normal leukapheresisderived $\mathrm{CD}_{34}{ }^{+}$cells transduced with shRUNX1 at days 6 and 7 of culture in order to detect RUNX1 targets involved in late stages of $\mathrm{MK}$ differentiation and, more particularly, in proplatelet formation and platelet function. A significant increase in 43 genes and a decrease in 61 genes was shown in both FPD/AML and shRUNX1-transduced MK (Figure $1 \mathrm{~A}$ and $\mathrm{B}$ and Online Supplementary Table S3A-F). Analysis of up-regulated genes did not show any potential candidates for FPD/AML platelet dysfunction (Online Supplementary Table S4A and Online Supplementary Figure S1). In contrast, analysis of GO pathways revealed two down-regulated genes, TREML1 and ITGA2, that were of interest regarding their role in platelet biology (Figure $1 \mathrm{C}$ and D and Online Supplementary Table S4B).

TREML1 codes for TLT-1, which represents an immunoreceptor tyrosine-based inhibition motif (ITIM)containing receptor exclusively expressed in MK and platelets, where it is stored in $\alpha$-granules. ${ }^{14}$ It undergoes surface translocation upon platelet activation ${ }^{14}$ and has been recently proposed to represent a more rapid and sensitive marker of platelet activation compared to $P$-selectin. ${ }^{15}$ TLT-1 ectodomain is released following platelet activation, leading to a naturally occurring soluble fragment (sTLT-1), which represents an abundant constituent of the platelet sheddome. ${ }^{16}$ Unlike other platelet ITIM receptors, the non-canonical TLT-1 has activating effects. ${ }^{17}$ Early work showed that, in a transiently transfected RBL-2H3 cell line, TLT-1 acts as a co-stimulatory receptor enhancing FceRI-mediated calcium signaling through recruitment of Src homology 2 domain-containing tyrosine phosphatase (SHP)-2 to its cytoplasmic ITIM domain. ${ }^{18}$ In human platelets, incubation with a blocking anti-TLT-1 antibody was shown to inhibit platelet aggregation triggered by thrombin, ${ }^{19}$ whereas, conversely,
sTLT-1 enhances platelet aggregation triggered by a variety of classic platelet agonists. ${ }^{17}$ Fibrinogen represents the only known ligand for TLT-1 and has been shown to bind both the full-length protein as well as the soluble form, ${ }^{17}$ and to favor fibrinogen deposition in vivo in a murine model of acute lung injury. ${ }^{20}$ Although the precise mechanism of TLT-1 action still has to be clearly defined, it has been proposed that, during platelet aggregation, fibrinogen is cross-linked by TLT-1, facilitating platelet-fibrinogen interactions and higher-order platelet aggregates, in concert with GPIIbIIIa. ${ }^{17}$ In addition, TLT-1 has been shown to interact through its cytoplasmic domain with ERM (ezrin/radixin/moesin) proteins, potentially linking fibrinogen to the platelet cytoskeleton. ${ }^{17}$ The essential role of TLT-1 in platelet function is revealed in Trem $/ 1^{-1-}$ mice, which display mild thrombocytopenia, decreased platelet aggregation, and prolonged bleeding time. ${ }^{17}$ In addition to its role in hemostasis, sTLT-1 released by platelets reduces inflammation and organ damage during sepsis by counteracting leukocyte activation and platelet-neutrophil crosstalk. ${ }^{21}$

ITGA2 encodes the $\alpha 2$ subunit of collagen receptor $\alpha 2 \beta 1$, which, in concert with GPVI, mediates the plateletcollagen interaction at sites of vascular injury required for stable platelet adhesion. ${ }^{22}$ Integrin $\alpha 2 \beta 1$ is essential when platelets are exposed to monomeric collagen, whereas it plays a supportive role for fibrillar collagen, where GPVI is the central receptor. The complementary interplay between both collagen receptors is required for an optimal platelet response to collagen. Upregulation of ITGA2 was demonstrated in K562 cells transduced with a RUNX1 expression vector, suggesting RUNX1 may regulate ITGA2 expression. ${ }^{23}$

Thus, we next assessed TREML1 and ITGA2 expression profile during in vitro megakaryopoiesis and showed that TREML1 and ITGA2 mRNA levels increase during normal MK differentiation (Figure 1E). Using real-time PCR, we confirmed that TREML1 and ITGA2 mRNAs are decreased in mature MK after shRNA-mediated RUNX1 inhibition (Figure 1F). Moreover, transduction of a WT RUNX1 cDNA carrying a mutation that does not change the amino acid sequence but prevents its recognition by shRUNX1 (RUNX1 ${ }^{\text {mut }}$ ) was able to rescue the inhibition in TREML1 and ITGA2 induced by shRUNX1 (Figure 1G), further demonstrating the relationship between RUNX1 and both TREML1 and ITGA2. 


\section{TREML1 and ITGA2 are novel RUNX1 targets}

To investigate whether TREML1 and ITGA2 represent direct RUNX1 targets, we searched for RUNX1 and activating histone mark $\mathrm{H} 3 \mathrm{~K} 27 \mathrm{Ac}$ enrichment across the entirety of these two genes by ChIP-sequencing in mature MK. Although no significant RUNX1 enrichment was shown in promoter regions, it was detected in intronic regions of both genes (Figure 2A and C). RUNX1 putative binding sites overlapping H3K27Ac were then identified by in silico analysis. Using ChIP, we confirmed 3-4-fold enrichment for RUNX1 in two RUNX1 sites identified in TREML1 and more than 5-fold enrichment in four sites identified in ITGA2 (Figure 2B and D). To assess whether these sites are functional, we cloned these intragenic regulatory regions (TREML1_RR and ITGA2_RR) upstream of TREML1 and ITGA2 promoters into lentivirus allowing mCherry reporter expression under these promoters (Online Supplementary Figure S2A and B) and tested them in
A

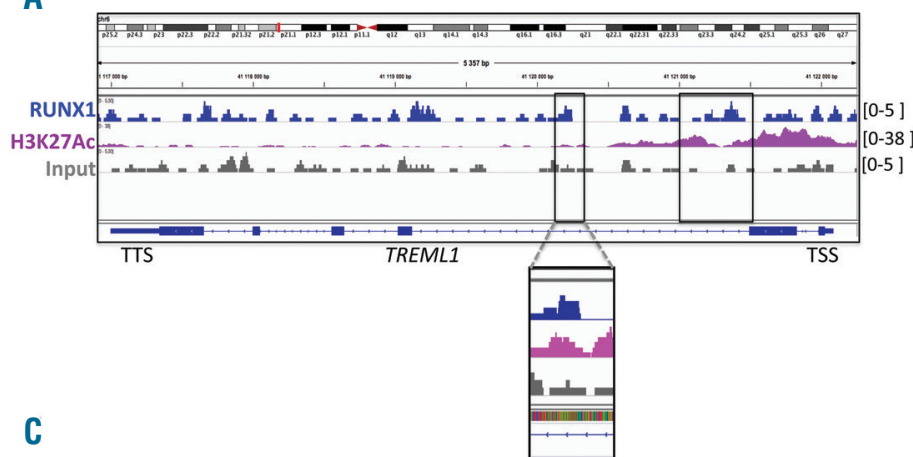

B

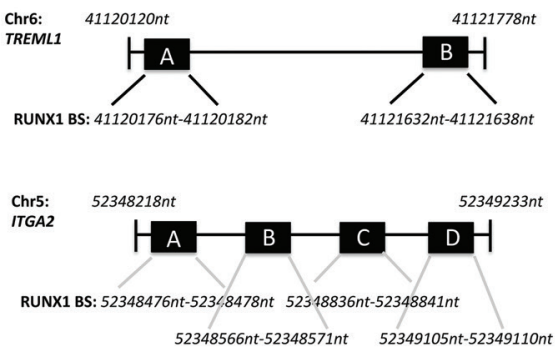

D

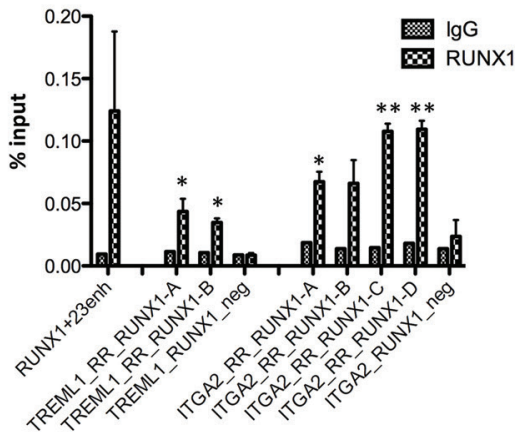

$\mathrm{E}$

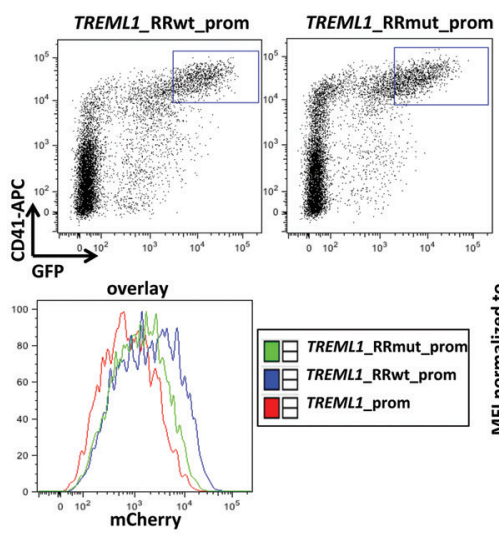

$\mathbf{F}$

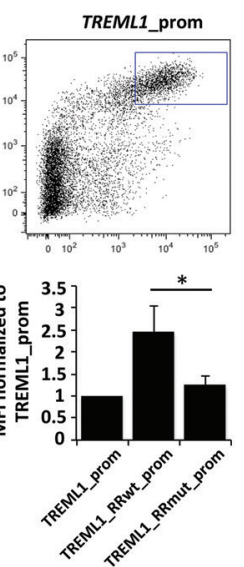

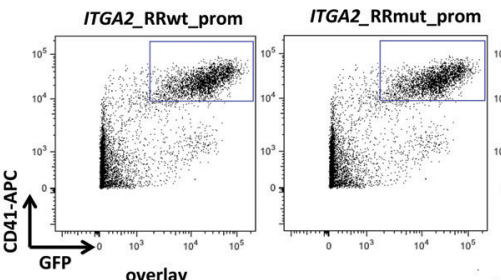

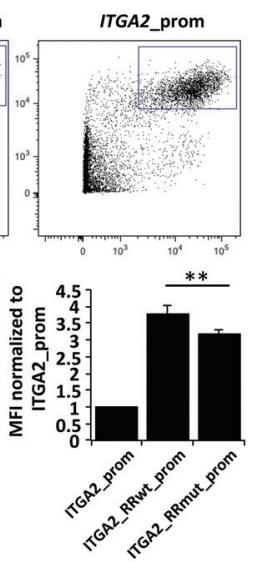

Figure 2. TREML1 and ITGA2 are novel RUNX1 targets. (A and C) Illustration of chromatin immunoprecipitation (ChIP)-sequencing peaks across TREML1 and ITGA2 genes in primary megakaryocytes (MK) differentiated from CD34+ cells isolated from leukapheresis samples (RUNX1 and H3K27Ac). (B and D) ChIP-polymerase chain reaction (PCR) assays performed in primary MK confirming that RUNX1 directly binds TREML1 and ITGA2 intragenic regions. Genomic location of putative RUNX1 binding sites (RUNX1 BS) based on the University of California Santa Cruz (UCSC) database (CGCh37/hg19). ChIP experiments show RUNX1 binding in both (A) and (B) RUNX1 BS identified in the intragenic region of the TREML1 gene and in (A-D) RUNX1 BS identified in the intragenic region of the ITGA2 gene. RUNX1+23 enhancer was used as a positive control. Amplification of regions without RUNX1 putative BS was performed as a negative control. Data represent Mean \pm Standard Deviation (SD) of three independent experiments; ${ }^{*} P<0.05 ; * * P<0.01$. (E and F) Functional analysis of identified RUNX1 BS. Intragenic TREML1 and ITGA2 regulatory regions were cloned upstream of TREML1 and ITGA2 promoters (RRwt_prom), respectively, in lentivirus allowing mCherry expression under these promoters and also encoding a PGK-GFP cassette, which allows selection of transduced MK. Mutagenesis was performed to delete all RUNX1 BS (RRmut_prom). MK were transduced on day 6 of culture and analyzed on day 10. (E) Results for TREML1. (F) Results for ITGA2. An identical gate was set for all conditions for each gene, including cells transduced with lentivirus carrying the promoter alone (right panel) or coupled with the wild-type (left) and mutated (middle) regulatory regions. For TREML1, Cherry expression was assessed in GFP-high CD41+ cells. Representative histograms are shown for one of four independent experiments. Median mCherry fluorescence intensity (MFI) was calculated relative to that obtained for ITGA2 prom $(n=4)$ and TREML1 prom $(n=4)$ constructs, respectively. Data represent Mean \pm SD of four independent experiments; $* P<0.05 ; * * P<0.01$ 
mature MK. Expression of mCherry fluorescent protein increased in both cases when TREML1_RR and ITGA2_RR were cloned upstream of the respective promoters and significantly decreased after specific deletion of RUNX1 binding sites (Figure $2 \mathrm{E}$ and F). These results clearly demonstrate that the identified sites are functional in mature $\mathrm{MK}$ and that TREML1 and ITGA2 are direct RUNX1 targets positively regulated by this transcription factor. mCherry reporter expression driven by TREML1_RR and ITGA2_RR harboring deleted RUNX1 sites did not reach the same level as for promoters alone, especially for ITGA2; this is probably because RUNX1 cooperates with other transcription factors. Indeed, ETS and
EVI1 binding sites and EVI1, SCL and ETS sites are identified in TREML1_RR and ITGA2_RR regions, respectively. Moreover, at least one other site positive for H3K27Ac mark binds RUNX1 at position 52362118-52362307nt (Figure 2C). This site could also be involved in the regulation of ITGA2 by RUNX1.

TLT-1 is decreased in platelets and megakaryocytes in familial platelet disorder with predisposition to acute myelogenous leukemia

Considering that the main role of TLT-1 is related to platelet function, we next assessed the levels in platelets. First, we showed decreased TREML1 transcripts by qPCR

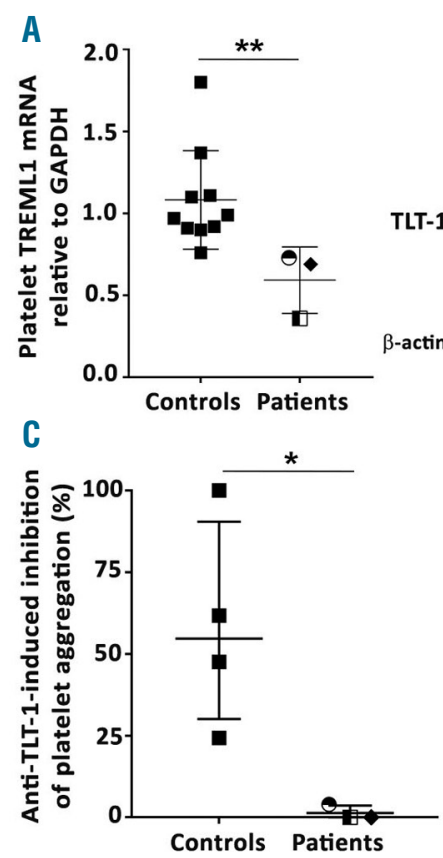

D

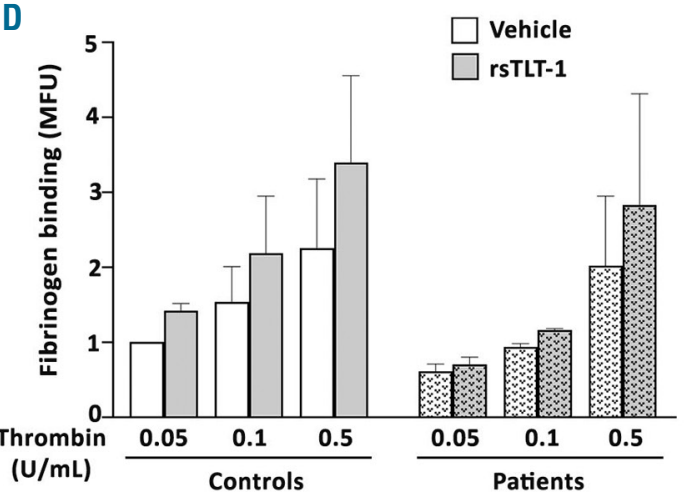

B
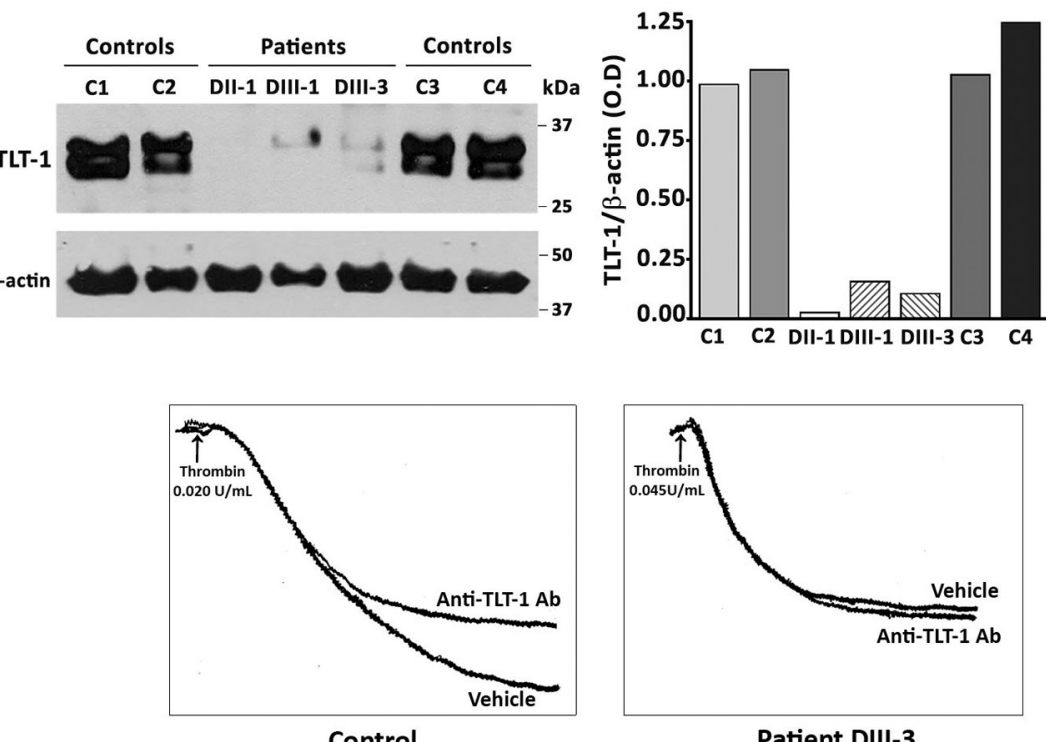

Control

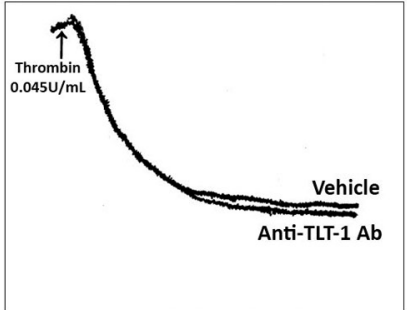

Patient DIII-3
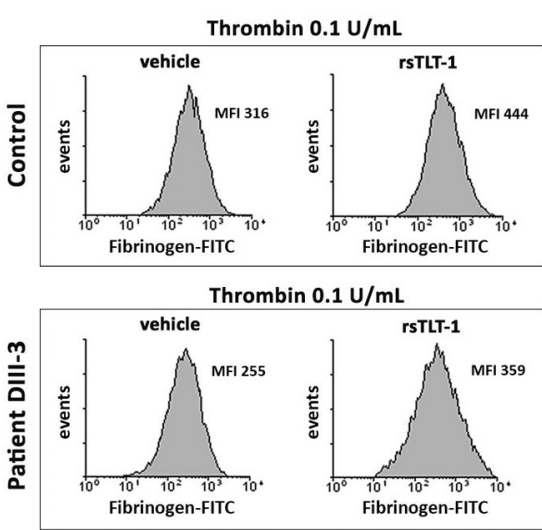

Figure 3. Levels of TLT-1 in familial platelet disorder with predisposition to acute myelogenous leukemia (FPD/AML) patient samples. (A) Real-time polymerase chain reaction (PCR) analysis of TREML1 transcript levels normalized to glyceraldehyde 3-phosphate dehydrogenase (GAPDH) in platelets from patients of pedigree D harboring the T219Rfs $* 8$ mutation ( $\mathrm{n}=3$ ): DII-1 $(\boldsymbol{\theta})$, DIII-1 $(\mathbf{D})$, DIII-3 $(*)$, and healthy subjects $(\mathrm{n}=10) ; * * P<0.01$. (B) (Left) Western blot analysis of TLT-1 content in platelet lysates from patients $(n=3)$ and controls $(n=4)$. Membranes were probed with anti-human TLT-1 antibody and reprobed with anti-beta ( $(\beta)$-actin. (Right) Optical density (OD) measurement of TLT-1/ $\beta$-actin ratio by densitometry. (C) Anti-TLT-1 antibody-induced inhibition of platelet aggregation. Patient ( $\mathrm{n}=3$ ): DII-1 $(\bullet)$, DIII-1 $(\mathbf{D})$, DIII-3 $\bullet$, and control $(n=4)$ washed platelets were incubated with an anti-TLT-1 blocking antibody $(A b)$ or vehicle, then challenged with thrombin and platelet aggregation was recorded in a Lumi-Aggregometer. Percentage inhibition in platelet aggregation induced by the anti-TLT-1 Ab was calculated relative to the vehicle (set as $100 \%$ ). Median values and interquartile range are shown; $* P<0.05$. (Right) Representative examples of aggregation traces of control and patient (DII-1) platelets incubated with anti-TLT-1 Ab or vehicle and challenged with thrombin. As patient platelets had reduced response to thrombin, thrombin concentration was titrated until a response was achieved. (D) Potentiation of thrombin-induced fibrinogen binding by recombinant soluble ( $r s)$ TLT-1. Patient $(n=2)$ and control $(n=2)$ washed platelets were incubated with rsTLT-1 or vehicle and exposed to increasing concentrations of thrombin. Mean fluorescence intensity (MFI) is expressed as arbitrary units (MFU). Median values and range are shown. (Right) Representative histograms and corresponding MFI values. 
in platelets from a third pedigree harboring the T219Rfs*8 mutation (pedigree D) (Figure 3A). Western blot analysis of platelet samples confirmed a marked decrease in TLT-1 content (Figure $3 \mathrm{~B}$ ), revealing for the first time downregulation of a member of the family of ITIM-bearing receptors in patients with RUNX1 mutations. We have previously shown that patients from this pedigree display partial deficiency of $\alpha$-granules, ${ }^{8}$ where TLT- 1 is located, and this finding was associated with a mild decrease in $\alpha$-granule protein TSP-1. ${ }^{8}$ Heterogeneous platelet content of other $\alpha$-granule proteins was demonstrated by other authors, including reduced levels of PF4, which is regulated by RUNX1, ${ }^{24}$ and preserved levels of $\beta$-TG and PDGF. ${ }^{25}$ More recently, RUNX1 deficiency has been linked to abnormal ER-to-Golgi trafficking and protein sorting leading to low von Willebrand factor (vWF) $\alpha$-granule content. ${ }^{26}$ Although down-regulated TREML1 gene expression secondary to RUNX1 loss-of-function seems to be the major mechanism leading to the low TLT-1 found in this study, we cannot exclude the possibility that $\alpha$ granule defects could contribute in part to the profound reduction in TLT-1 protein.

Whereas incubation of normal platelets with a blocking anti-TLT-1 antibody inhibited thrombin-induced platelet aggregation, as previously reported, ${ }^{19}$ it had no effect on thrombin-induced aggregation of FPD/AML platelets (Figure 3C), further demonstrating the absence of relevant amounts of TLT-1 in patient platelets. Conversely, consistent with the functional role of sTLT-1 in platelet activation, ${ }^{17}$ and as previously shown for platelet adhesion and actin polymerization on fibrinogen matrices, ${ }^{27}$ a recombinant soluble fragment (rsTLT-1) was able to potentiate thrombin-induced fibrinogen binding to normal platelets (Figure 3D). In the absence of rsTLT-1, thrombin-induced fibrinogen binding was lower in patients from pedigree $\mathrm{D}$ compared to controls, as previously reported. ${ }^{8}$ This defect was partially corrected by incubation of patient platelets with rs TLT-1 (Figure 3D), pointing to a role for TLT-1 deficiency in the platelet function defect. However, several other abnormalities are also involved, possibly contributing to the variability in platelet aggregation tests among patients (Table 1).

As shown in this work, TREML1 gene expression levels are low in normal early megakaryopoiesis and increase markedly along $\mathrm{MK}$ maturation reaching higher expression levels in mature MK. Double immunofluorescence labeling of TLT-1 and $\mathrm{vWF}$ in mature $\left(\mathrm{CD} 41^{+} \mathrm{CD} 42^{+}\right) \mathrm{MK}$ from one control and 2 patients (DII-1 and DIII-3) confirmed that TLT-1 is mainly localized in $\alpha$-granules (Figure 4 ), as previously reported. ${ }^{14}$ However, part of TLT-1 does not co-localize with vWF and is present in a different subpopulation of $\alpha$-granules. Interestingly, a distinct staining pattern of TLT1 and $P$-selectin was recently shown in mouse and human platelets and mouse $M K$, suggesting differential compartmentalization of these proteins within $\alpha$-granules, ${ }^{15}$ as previously shown for other proteins packaged in platelets. ${ }^{28}$ TLT-1-positive granules were less abundant or absent in patient compared to control MK (Figure 4).

The role of TLT-1 in normal MK is not known. TLT-1deficient mice display a $20 \%$ decrease in platelet counts, ${ }^{17}$ although the underlying mechanism has not been explored. In order to gain insight into this, we incubated human cord blood or leukapheresis-derived MK with a blocking anti-TLT-1 antibody or a control IgG. There was no significant difference in megakaryocyte output and maturation in the presence of anti-TLT-1 compared to control, whereas proplatelet formation was reduced (Online Supplementary Figure S3), suggesting TLT-1 may have a role in normal proplatelet formation. However, further study will be required to definitively establish this issue and to determine whether TLT-1 deficiency contributes to defective platelet production in FPD/AML.

Levels of integrin subunit $\alpha 2$ and collagen adhesion are decreased in platelets in familial platelet disorder with predisposition to acute myelogenous leukemia

Transcript levels of ITGA2, coding for the $\alpha 2$ integrin subunit of collagen receptor $\alpha 2 \beta 1$, were also shown to be decreased in platelets from pedigree $\mathrm{D}$ by qPCR (Figure $5 \mathrm{~A}$ ). Accordingly, surface expression of $\alpha 2$ (GPIa) was substantially reduced, as revealed by analysis of platelet-rich plasma (Figure 5B) and whole blood flow cytometry (Online Supplementary Table S5), and further confirmed by western blot (Figure 5C). The reduction in surface $\alpha 2$ was associated with a decrease in platelet surface expression of the heterodimeric $\beta 1$ subunit (GPIIa), whereas GPVI, GPIIbIIIa, GPIb-IX were preserved (Table 2), indicating a selective abnormality in the $\alpha 2 \beta 1$ complex. The reduction in $\beta 1$ is probably due to the concomitant decrease in its $\alpha 2$ partner, as $\beta 1$ subunit (ITGB1) mRNA levels were preserved (Online Supplementary Figure S4). In addition, platelet surface levels of $\alpha 5$ and $\alpha 6$ integrin subunits, which also heterodimerize with $\beta 1$, were normal or in the lower normal limit (Table 2).

Table 2. Surface platelet glycoprotein expression.

\begin{tabular}{|c|c|c|c|c|c|c|c|c|}
\hline \multirow[t]{2}{*}{ Patient } & \multicolumn{7}{|c|}{ Relative filuorescence intensity (patient/control ratio) } & \multirow[b]{2}{*}{$\alpha 6$ integrin } \\
\hline & GPlla ( $\beta 1)$ & GPVI & GPIlb & GPIIla & GPlb & GPIX & $\alpha 5$ integrin & \\
\hline AII-1 & 0.48 & nd & 0.98 & 1.09 & 1.44 & 1.22 & nd & nd \\
\hline AII-2 & 0.65 & nd & 0.96 & 1.07 & 1.87 & 1.47 & nd & nd \\
\hline BII-2 & nd & nd & 0.90 & 1.01 & 1.99 & 1.85 & nd & nd \\
\hline BIII-1 & nd & nd & 0.94 & 0.97 & 2.37 & 2.07 & nd & nd \\
\hline DII-1 & 0.43 & 0.79 & 0.79 & 1.02 & 1.51 & 1.84 & 0.95 & 0.78 \\
\hline DIII-1 & 0.77 & 0.89 & 0.79 & 0.91 & 1.42 & 1.74 & 1.29 & 1.32 \\
\hline DIII-3 & 0.65 & 0.80 & 0.98 & 1.03 & 1.58 & 1.28 & 0.98 & 0.78 \\
\hline Ref. range & 0.81-1.19 & $0.73-1.27$ & $0.78-1.22$ & $0.78-1.22$ & $0.65-1.35$ & $0.81-1.19$ & $0.85-1.12$ & $0.70-1.55$ \\
\hline
\end{tabular}

GP: glycoprotein. Ref.; reference; nd; not done. GPIIa represents the $\beta 1$ integrin subunit of the collagen receptor $\alpha 2 \beta 1$. Relative fluorescence intensity (RFI) was calculated as the ratio between the corresponding antibody and the isotypic control. Results are expressed as the ratio between RFI in each patient and a simultaneously assayed control sample. The reference range was established by the Mean \pm two Standard Deviations of ten healthy subjects who were simultaneously studied. 

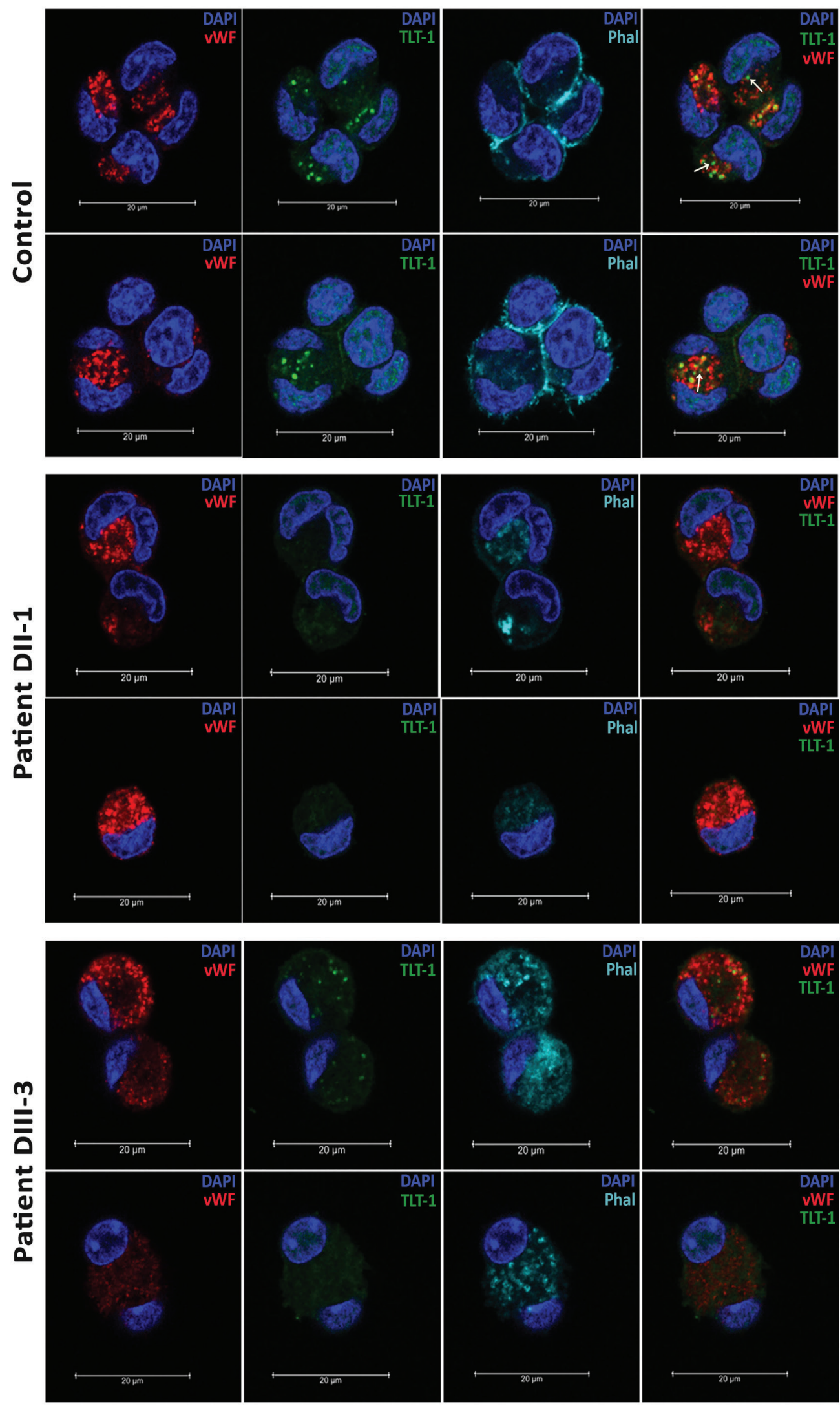

Figure 4. Levels of TLT-1 in megakaryocytes (MK) in familial platelet disorder with predisposition to acute myelogenous leukemia. Double immunofluorescence labeling of TLT-1 and von Willebrand factor (VWF) in mature MK grown from peripheral blood CD34+ cells from one control and 2 patients (DII-1 and DIII-3). Representative confocal microscopy images of sorted CD41+D42+ cells stained with rabbit anti-TLT-1, mouse anti-vWF, Phallodin-Alexa 633 and 4',6-diamidino-2 phenylindole. Anti-rabbit Alexa-488 and anti-mouse Alexa-546 were used as secondary antibodies. White arrows indicate staining for TLT-1 in granules that do not contain vWF. 
Low $\alpha 2 \beta 1$ has been described in ANKRD26-related thrombocytopenia (RT), ${ }^{29}$ which shares several features with FPD/AML. However, whereas we confirmed that reduced $\alpha 2$ was restricted to some but not all ANKRD26RT patients (Online Supplementary Figure S5), all FPD/AML patients studied showed this defect. Interestingly, $\alpha 2$ defi- ciency was recently reported in two other FPD/AML pedigrees. ${ }^{30,31}$ Study of a larger cohort is required to determine whether low $\alpha 2$ is a constant feature of FPD/AML and could be useful as a screening tool in this setting.

Consistent with the essential role of $\alpha 2 \beta 1$ in platelet interaction with monomeric collagen $I_{,}^{22,32}$ patient platelet
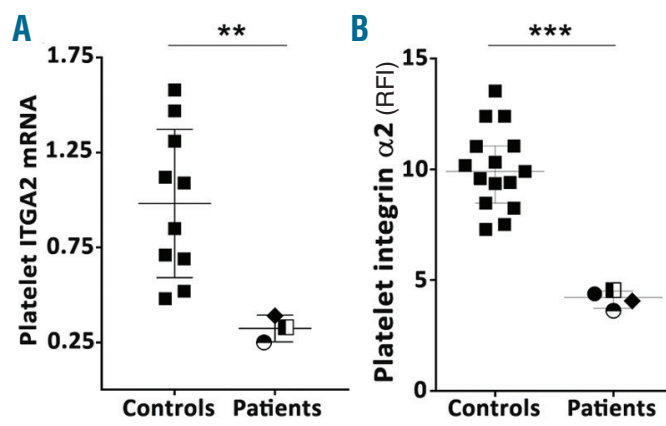

$\mathbf{D}$

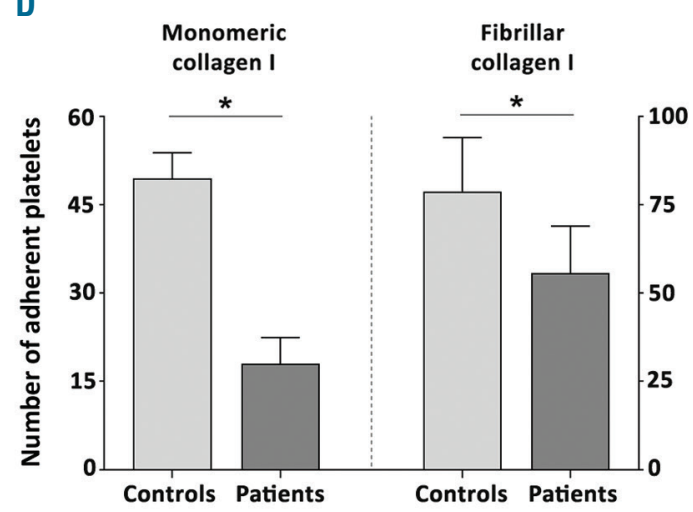

C

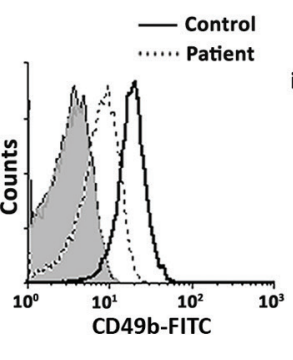

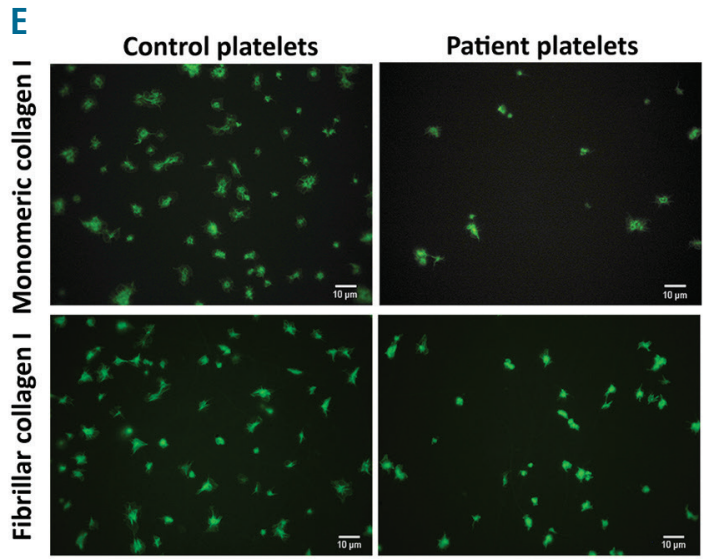

$\mathbf{F}$
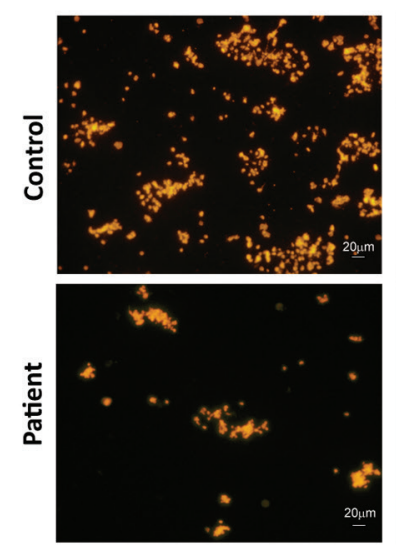

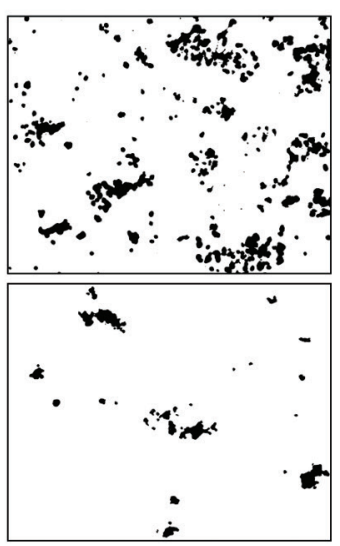

G

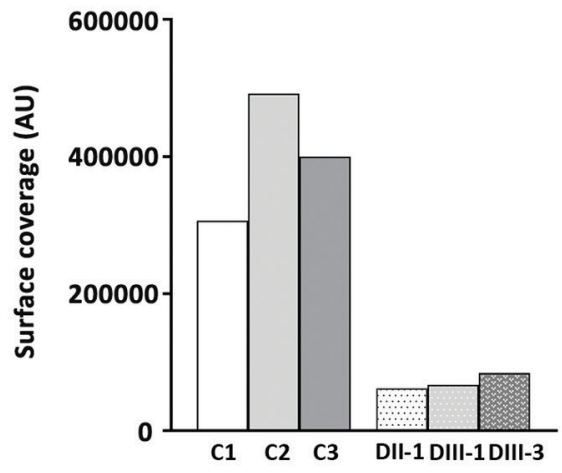

Figure 5. Levels of integrin subunit alpha $(\alpha)-2$ and collagen adhesion in platelets in familial platelet disorder with predisposition to acute myelogenous leukemia (FPD/AML). (A) Real-time polymerase chain reaction (PCR) analysis of ITGA2 transcript levels normalized to GAPDH in patients from pedigree D harboring the T219Rfs $* 8$ mutation $(n=3)$ : DII-1 $(\boldsymbol{\theta})$, DIII-1 $(\mathbf{D})$, DIII-3 $(*)$, and healthy subjects $(n=10) ; * P<0.01$. (B) (Left) Platelet surface levels of integrin subunit alpha $(\alpha)-2$ by flow cytometry. Results are expressed as the ratio between fluorescence intensity obtained with anti-CD49b antibody and the corresponding isotype control (relative fluorescence intensity) for patients $(n=4)$ : DII-1 $(\boldsymbol{\theta})$, DIII-1 $(\mathbf{D})$, DIII-3 $\bullet$, All-1 $(\bullet)$ and controls $(n=15)$. Median values and interquartile range are shown; $* * * P<0.001$. (Right) Representative histograms of integrin $\alpha 2$ in patient (dashed line) and control (thick continuous black line) platelets. Isotype controls in the patient and control are shown by superimposed solid gray and empty gray histograms, respectively. (C) Western blot analysis of platelet integrin subunit $\alpha 2$. Platelet lysates from patients $(n=3)$ and controls $(n=4)$ were subjected to sodium dodecyl sulphate-polyacrylamide gel electrophoresis (SDS-PAGE); membranes were probed with anti-human integrin $\alpha 2$ antibody and reprobed with anti-beta ( $\beta$ )-actin. (Bottom) Densitometric analysis of integrin $\alpha 2 / \beta$-actin ratio. (D) Platelet adhesion to monomeric and fibrillar type I collagen in patients ( $n=3$ : DII-1; DIII-1; DIII-3) and controls $(n=5)$. Platelets were stained with Phalloidin-fluorescein isothiocyanate (FITC) labeled peptide, and the number of adherent platelets per field at 1000x magnification was counted. Bars represent median values and interquartile range; $* P<0.05$. (E) Representative images of platelet adhesion to monomeric and fibrillar collagen are shown. ( $F$ and $G$ ) Platelet accumulation under flow. Platelets from controls $(n=3)$ and patients $(n=3)$ were perfused over fibrillar collagen l-coated coverslips through a flow chamber at 1 dyn/ $\mathrm{cm}^{2}$, labeled with phalloidin-tetramethylrhodamine and platelet surface coverage was determined using Image J software. (F) Representative images of control and patient platelets obtained at 400x magnification are shown. AU: arbitrary units. 
adhesion to this substrate was severely impaired (Figure $5 \mathrm{D}$ and $\mathrm{E}$ ). Moreover, platelet adhesion was also, albeit less markedly, reduced over fibrillar collagen I (Figure 5D and E). Considering that GPVI, which has a more prominent role over fibrillar collagen, was preserved, other abnormalities in FPD/AML platelets may contribute to defective adhesion to fibrillar collagen in addition to low $\alpha 2 \beta 1$. In contrast, platelet adhesion to convulxin, which relies on GPVI, and to fibrinogen, which depends mainly on GPIIbIIIa, were largely preserved (Online Supplementary Table S6). As an approach to mimic the in vivo conditions, we studied platelet aggregate formation under flow, which revealed a substantial decrease in surface area coverage over a collagen substrate for patient compared to control platelets (Figure 5F and G). Overall, these abnormalities suggest that primary hemostasis might be impaired in FPD/AML, although the clinical relevance of this finding still has to be determined. On the other hand, defective collagen-induced aggregation (Table 1) cannot be explained only by decreased $\alpha 2 \beta 1$, as collagen preparations used in aggregometry depend mainly on GPVI. Given this, the aggregation defect in response to collagen and other platelet agonists may also rely on the role played by TLT-1 in fibrinogen binding, platelet aggregate formation and stabilization.

\section{Megakaryocytes in familial platelet disorder with predisposition to acute myelogenous leukemia display low levels of $\alpha 2$ integrin subunit and decreased adhesion to collagen I}

Platelet production is a tightly regulated process governed by the close interaction between $\mathrm{MK}$ and bone marrow (BM) extracellular matrix (ECM) proteins. In addition to its abundance at the vascular bed, collagen I is a crucial component of the BM ECM. MK-collagen I interaction is of critical importance in restraining proplatelet formation at the osteoblastic niche, thus preventing premature platelet release into the interstitial space and allowing normal platelet production into the lumen of BM sinusoids. ${ }^{33}$ Ligation of the $\alpha 2 \beta 1$ receptor in $\mathrm{MK}$ is required for stress fiber formation and adhesion over collagen I, as shown in both human and mouse $M K,{ }^{33,34}$ whereas although $\alpha 2 \beta 1$ integrin is involved in collagen I-induced inhibition of proplatelet formation in human $M K,{ }^{33}$ it does not seem to be essential in mouse MK, where GPVI mediates the inhibitory signal. ${ }^{34}$ Considering the key function of $\alpha 2 \beta 1$ in $M K$ behavior over collagen, we next studied MK $\alpha 2$ surface expression and found decreased levels on patient mature $\left(\mathrm{CD} 41^{+} \mathrm{CD} 42^{+}\right) \mathrm{MK}$ (Figure 6A). There was a trend towards reduced $\mathrm{MK}\left(\mathrm{CD} 41^{+}\right)$adhesion to fibrillar type I collagen, which was confirmed after sorting the mature $\left(\mathrm{CD} 41^{+} \mathrm{CD} 42^{+}\right) \mathrm{MK}$ population, whereas adhesion to fibrinogen was preserved (Figure $6 \mathrm{~B}$ and $\mathrm{C}$ ). In addition to low $\alpha 2$ levels, other defects in FPD/AML MK could contribute to reduced collagen adhesion. Virtual absence of proplatelet formation from patient cells ${ }^{2}$ prevented us from assessing whether physiological collagen I-inhibition in thrombopoiesis was affected. The role of $\alpha 2 \beta 1$ in platelet production in vivo remains controversial as, unexpectedly, Itga $2^{-/}$mice show normal platelet counts. ${ }^{32}$ Increased MK numbers found in these mice may represent a compensatory mechanism and provide an explanation for the absence of thrombocytopenia in this model. We have previously shown that FPD/AML patients show decreased $M K$ output from hematopoietic progenitors,
A

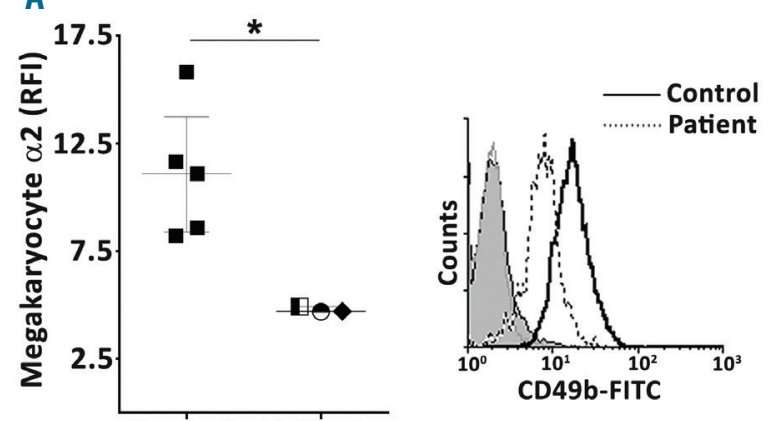

Controls Patients

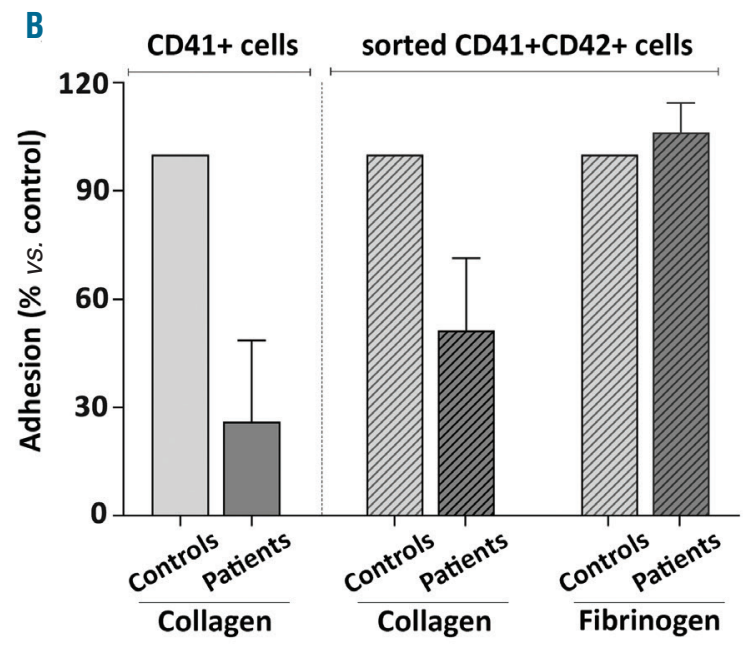

C
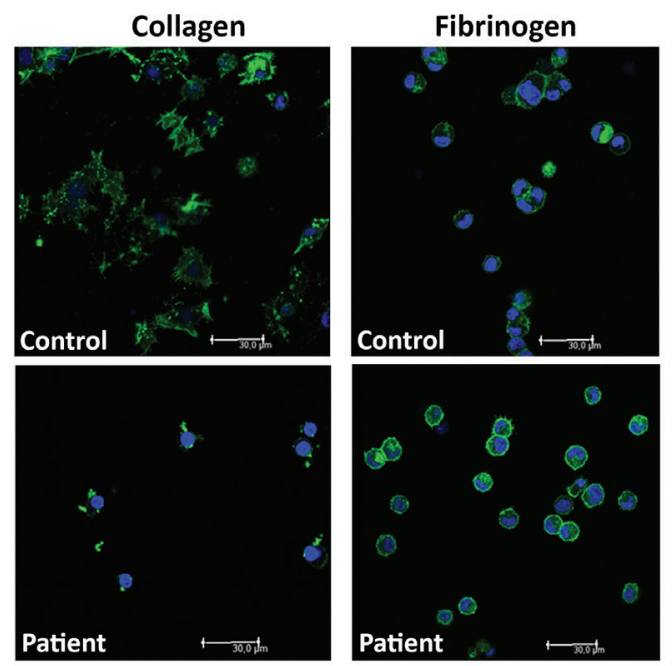

Figure 6. Levels of integrin subunit alpha $(\alpha)-2$ and collagen adhesion in megakaryocytes (MK) in familial platelet disorder with predisposition to acute myelogenous leukemia (FPD/AML). (A) (Left) Flow cytometry analysis of integrin $\alpha-2$ expression in mature $\left(C D 41^{+} C D 42^{+}\right)$MK grown from peripheral blood CD34 cells from patients ( $\mathrm{n}=3)$ : DII-1 $(\boldsymbol{\theta})$, DIII-1 $\left(\mathbf{\mathbf { D }}^{+}\right)$, DIII-3 $(\bullet)$, and healthy subjects $(n=5)$. Median relative fluorescence intensity values and interquartile range are depicted; $* P<0.05$. (Right) Representative histograms of CD $49 \mathrm{~b}$ expression in patient (dashed line) and control (thick continuous black line) MK. Isotype controls in the patient and control are shown by superimposed solid gray and empty gray histograms, respectively. (B) MK adhesion to fibrillar type I collagen and fibrinogen in patients. The number of adherent cells was expressed as percentage of simultaneously assayed controls, set as 100\%. (Left) Results for MK (CD41 ${ }^{+}$) adhesion to collagen in patients ( $n=3$ : DII-1, DIII-1 and DIII-3). In separate experiments, $C D 41^{+} C D 42^{+}$cells were sorted by flow cytometry $(n=2$ : DIII-3 and BIII-1), allowed to adhere to collagen or fibrinogen-coated surfaces; (middle and right) results are shown. Bars represent median values and interquartile range; $P=$ not significant. (C) Representative images of patient and control adherent mature $\left(C D 41^{+} C D 42^{+}\right)$MK stained with phalloidin-fluorescein isothiocyanate (FITC) and 4',6-diamidino-2-phenylindole. 
delayed MK maturation, and low ploidy levels. ${ }^{2}$ On this basis, it seems reasonable to consider the possibility that, in vivo, FPD/AML MK may not be able to fully compensate for the decrease in $\alpha 2$ and, in this scenario, $\alpha 2$ downregulation could possibly contribute to impaired platelet production in patients in addition to the above-mentioned MK abnormalities.

Considering that FPD/AML is a heterogeneous condition, study of additional pedigrees would be useful to determine whether patients harboring $R U N X 1$ mutations different from those included in this study display similar defects.

In conclusion, gene expression analysis of RUNX1 knock-down or mutated MK proved to be a suitable approach to identify novel RUNX1 targets involved in platelet biology. TREML1 and ITGA2 may now be added to the growing list of genes regulated by RUNX1 in the megakaryocytic lineage. Down-regulated expression of these genes in patients contributes to the platelet defects induced by RUNX1 mutations. These findings highlight the key role of RUNX1 as a master regulator of the MK lineage, where it modulates the expression of a diverse array of genes crucial to platelet production and function. They also help further unravel the molecular mechanisms underlying the FPD/AML phenotype.

\section{Funding}

This study was supported by a French grant from the Ligue National Contre le Cancer (LNCC, équipe labellisée to HR, 2013 and 2016), the European grant ERA-NET (to C. Balduini, 2013), the Argentinian grant from the National Agency for Scientific and Technological Research (to $P H, 2012)$, a grant from the Fondation Nelia et Amadeo Barletta (to PH, 2017) and the cooperation program between France and Argentina, Ecos-Sud-Ministerio de Ciencia, Tecnología e Innovación Productiva (MINCyT) (to HR and $P H, 2016)$. DS was supported by a postdoctoral fellowship from LNCC. The microarray was funded by the apprenticeship tax from Gustave Roussy, Villejuif, France.

\section{Acknowledgments}

We thank the patients and their families for participating in this study. We thank P. Rameau for flow cell sorting and flow cytometry analysis (PFIC, Gustave Roussy, Villejuif, France), G. Meurice for transcriptome analysis (UMS AMMICA, INSERM US23/CNRS UMS S3665, Gustave Roussy, Villejuif, France), Gabriel Correa (Instituto Lanari, University of Buenos Aires, Argentina) for coagulation studies, Mirta Schattner (IMEXCONICET, Buenos Aires, Argentina) for help with the flow chamber assay and Daniela Ayala (UE IDIM-CONICET) for help with Western blot experiments. We are sincerely grateful for the support of the Ferrata Storti Foundation.

\section{References}

1. Tijssen MR, Cvejic A, Joshi A, et al. Genome-wide analysis of simultaneous GATA1/2, RUNX1, FLI1, and SCL binding in megakaryocytes identifies hematopoietic regulators. Dev Cell. 2011;20(5):597-609.

2. Bluteau D, Glembotsky AC, Raimbault A, et al. Dysmegakaryopoiesis of FPD/AML pedigrees with constitutional RUNX1 mutations is linked to myosin II deregulated expression. Blood. 2012;120(13):27082718.

3. Zang C, Luyten A, Chen J, Liu XS, Shivdasani RA. NF-E2, FLI1 and RUNX1 collaborate at areas of dynamic chromatin to activate transcription in mature mouse megakaryocytes. Sci Rep. 2016;6:30255

4. Ichikawa M, Asai T, Saito T, et al. AML-1 is required for megakaryocytic maturation and lymphocytic differentiation, but not for maintenance of hematopoietic stem cells in adult hematopoiesis. Nat Med. 2004;10(3):299-304.

5. Schlegelberger B, Heller PG. RUNX1 deficiency (familial platelet disorder with predisposition to myeloid leukemia, FPDMM). Semin Hematol. 2017;54(2):7580.

6. Michaud J, Wu F, Osato M, et al. In vitro analyses of known and novel RUNX1/AML1 mutations in dominant familial platelet disorder with predisposition to acute myelogenous leukemia: implications for mechanisms of pathogenesis. Blood. 2002;99(4):1364-1372.

7. Antony-Debré I, Manchev VT, Balayn N, et al. Level of RUNX1 activity is critical for leukemic predisposition but not for thrombocytopenia. Blood. 2015;125(6):930-940.

8. Glembotsky AC, Bluteau D, Espasandin YR, et al. Mechanisms underlying platelet function defect in a pedigree with familial platelet disorder with a predisposition to acute myelogenous leukemia: potential role for candidate RUNX1 targets. J Thromb Haemost. 2014;12(5):761-772.

9. Latger-Cannard V, Philippe C, Bouquet A et al. Haematological spectrum and genotype-phenotype correlations in nine unrelated families with RUNX1 mutations from the French network on inherited platelet disorders. Orphanet J Rare Dis. 2016;11:49.

10. Sun L, Gorospe IR, Hoffman EP, Rao AK Decreased platelet expression of myosin regulatory light chain polypeptide (MYL9) and other genes with platelet dysfunction and CBFA2/RUNX1 mutation: insights from platelet expression profiling. J Thromb Haemost. 2007;5(1):146-154.

11. Heller PG, Glembotsky AC, Gandhi MJ, et al. Low Mpl receptor expression in a pedigree with familial platelet disorder with predisposition to acute myelogenous leukemia and a novel AML1 mutation. Blood. 2005;105(12):4664-4670.

12. Antony-Debré I, Bluteau D, Itzykson R, et al. MYH10 protein expression in platelets as a biomarker of RUNX1 and FLI1 alterations. Blood. 2012;120(13):2719-2722 .

13. Gilles L, Guièze R, Bluteau D, et al P19INK4D links endomitotic arrest and megakaryocyte maturation and is regulated by AML-1. Blood. 2008;111(8):4081 4091.

14. Washington AV, Schubert RL, Quigley L, et al. A TREM family member, TLT-1, is found exclusively in the alpha-granules of megakaryocytes and platelets. Blood. 2004; 104(4):1042-1047

15. Smith CW, Raslan Z, Parfitt L, et al. TREM like transcript 1: a more sensitive marker of platelet activation than P-selectin in humans and mice. Blood Adv. 2018;2(16):2072-2078.

16. Gattis JL, Washington AV, Chisholm MM et al. The structure of the extracellular domain of triggering receptor expressed on myeloid cells like transcript-1 and evidence for a naturally occurring soluble fragment. J Biol Chem. 2006;281(19):13396-13403.

17. Washington AV, Gibot S, Acevedo I, et al. TREM-like transcript-1 protects against inflammation-associated hemorrhage by facilitating platelet aggregation in mice and humans. J Clin Invest. 2009;119(6):14891501.

18. Barrow AD, Astoul E, Floto A, et al. Cutting edge: TREM-like transcript-1, a platelet immunoreceptor tyrosine-based inhibition motif encoding costimulatory immunoreceptor that enhances, rather than inhibits, calcium signaling via SHP-2.J Immunol. 2004;172(10):5838-5842.

19. Giomarelli B, Washington VA, Chisholm $M M$, et al. Inhibition of thrombin-induced platelet aggregation using human singlechain Fv antibodies specific for TREM-like transcript-1. Thromb Haemost. 2007; 97(6):955-963.

20. Morales-Ortíz J, Deal V, Reyes F, et al. TLT1 is a prognostic indicator in ALI/ARDS and prevents tissue damage in the lungs in a mouse model. Blood. 2018;132(23):24952505.

21. Derive M, Bouazza Y, Sennoun N, et al. Soluble TREM-like transcript-1 regulates leukocyte activation and controls microbial sepsis. J Immunol. 2012;188(11):5585-5592.

22. Nieswandt B, Watson SP. Platelet-collagen interaction: is GPVI the central receptor? Blood. 2003;102(2):449-461

23. Elagib KE, Racke FK, Mogass M, Khetawat R, Delehanty LL, Goldfarb AN. RUNX1 and GATA-1 coexpression and cooperation in megakaryocytic differentiation. Blood. 2003;101(11):4333-4341.

24. Aneja K, Jalagadugula G, Mao G, Singh A Rao AK. Mechanism of platelet factor 4 
(PF4) deficiency with RUNX1 haplodeficiency: RUNX1 is a transcriptional regulator of PF4. J Thromb Haemost. 2011; 9(2):383-391.

25. Weiss HI, Witte LD, Kaplan KL, et al. Heterogeneity in storage pool deficiency: studies on granule-bound substances in 18 patients including variants deficient in alpha-granules, platelet factor 4 , beta-thromboglobulin, and platelet-derived growth factor. Blood. 1979;54(6):1296-1319.

26. Jalagadugula G, Goldfinger LE, Mao G, Lambert MP, Rao AK. Defective RAB1Brelated megakaryocytic ER-to-Golgi transport in RUNX1 haplodeficiency: impact on von Willebrand factor. Blood Adv. 2018:2(7):797-806.

27. Morales J, Villa K, Gattis J, et al. Soluble TLT-1 modulates platelet-endothelial cell interactions and actin polymerization. Blood Coagul Fibrinolysis. 2010;21(3):229236.

28. Chatterjee M, Huang Z, Zhang W, et al Distinct platelet packaging, release, and surface expression of proangiogenic and antiangiogenic factors on different platelet stimuli. Blood. 2011; 117(14):3907-3911.

29. Noris P, Perrotta S, Seri M, et al. Mutations in ANKRD26 are responsible for a frequent form of inherited thrombocytopenia: analysis of 78 patients from 21 families. Blood. 2011;117(24):6673-6680.

30. Perez Botero J, Chen D, Cousin MA, et al. Clinical characteristics and platelet phenotype in a family with RUNX1 mutated thrombocytopenia. Leuk Lymphoma. 2017:58(8):1963-1967.

31. De Rocco D, Melazzini F, Marconi C, et al.
Mutations of RUNX1 in families with inherited thrombocytopenia. Am Hematol. 2017;92(6):E86-E88

32. Holtkötter O, Nieswandt B, Smyth N, et al Integrin alpha 2-deficient mice develop normally, are fertile, but display partially defective platelet interaction with collagen J Biol Chem. 2002;277(13):10789-10794

33. Sabri S, Jandrot-Perrus M, Bertoglio J, et al. Differential regulation of actin stress fiber assembly and proplatelet formation by alpha2beta1 integrin and GPVI in human megakaryocytes. Blood. 2004; 104(10): 3117-3125.

34. Semeniak D, Kulawig R, Stegner D, et al. Proplatelet formation is selectively inhibited by collagen type I through Syk-independent GPVI signaling. J Cell Sci. 2016 129(18):3473-3484. 